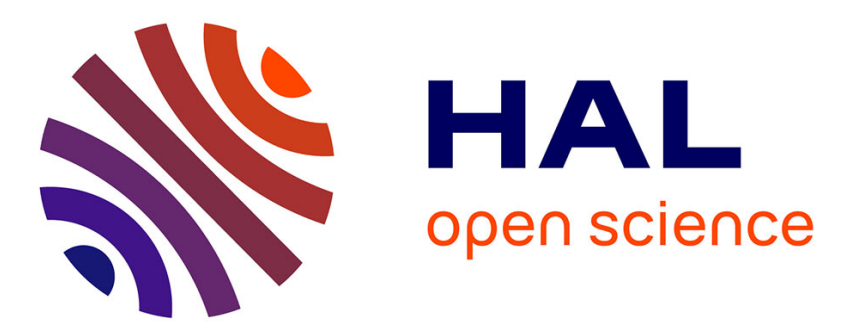

\title{
Toward modeling anisotropic yield stress and consistency induced by fiber in fiber-reinforced viscoplastic fluids
}

\author{
Julien Férec, Arnaud Perrot, Gilles Ausias
}

\section{To cite this version:}

Julien Férec, Arnaud Perrot, Gilles Ausias. Toward modeling anisotropic yield stress and consistency induced by fiber in fiber-reinforced viscoplastic fluids. Journal of Non-Newtonian Fluid Mechanics, 2015, 220, pp.69-76. 10.1016/j.jnnfm.2014.08.009 . hal-01113109

\author{
HAL Id: hal-01113109 \\ https://hal.science/hal-01113109
}

Submitted on 11 Sep 2018

HAL is a multi-disciplinary open access archive for the deposit and dissemination of scientific research documents, whether they are published or not. The documents may come from teaching and research institutions in France or abroad, or from public or private research centers.
L'archive ouverte pluridisciplinaire HAL, est destinée au dépôt et à la diffusion de documents scientifiques de niveau recherche, publiés ou non, émanant des établissements d'enseignement et de recherche français ou étrangers, des laboratoires publics ou privés. 


\title{
Toward modeling anisotropic yield stress and consistency induced by fiber in fiber-reinforced viscoplastic fluids
}

\author{
J. Férec *, A. Perrot, G. Ausias \\ Université de Bretagne-Sud, EA 4250, LIMATB, 56100 Lorient, France
}

\begin{abstract}
A model is developed for suspensions of rigid fibers in a non-Newtonian fluid exhibiting a yield stress by taking into account hydrodynamic and fiber-fiber interactions. To be applied to fiber-reinforced viscoplastic fluids such as cementitious pastes or mortars, the matrix behavior is described by a Herschel-Bulkley law. The model is able to predict shear thinning and shear thickening behaviors as well as anisotropic yield stress depending on the fiber orientation. The extra stress term involves structural tensors, where exact analytical solutions have been proposed for an isotropic fiber orientation in simple shear flow. The model predictions show a good agreement with the yield stress values of steel fibers dispersed in kaolin pastes.
\end{abstract}

\section{Introduction}

In the past decade, fiber-reinforced cementitious materials have become structural materials which can be more efficient and economical than common steel rebars reinforced concrete, for instance. Successful precast or cast-in-place applications, where fibers partially or totally supplement steel rebars, are reported in the literature [1-5]. Fiber-reinforced concrete precast segments or fiber-reinforced sprayed concrete or mortar are also technological solutions for tunneling or roofing. Consequently, design codes have recently been written in order to develop fiber-reinforced concretes as structural materials [6-8]. Addition of fibers into cementitious materials improves toughness, impact resistance and ductility, reduces the tendency to crack propagation, prevents spalling in case of fire loading and provides higher mechanical strength [9-20]. Therefore, fibers are used to ensure users safety and to design ultra-high performance fiber-reinforced concrete.

Fibers are generally included in a cementitious blend that is mixed and placed more or less conventionally. Knowing the flow properties of the fresh material is a key point as fibers are able to move and to orient due to the concrete flow. Therefore, fibers may induce anisotropic and heterogeneous mechanical behavior that depends strongly on their dispersion and orientation state $[13,21-26]$. It is well established that fiber orientation depends

\footnotetext{
* Corresponding author.

E-mail address: julien.ferec@univ-ubs.fr (J. Férec).
}

on both process and material rheology [5,9,10,13,15,22,27-32]. Then, some works have been carried out on the effect of fibers on the concrete rheology $[11,28,30,33-35]$ and on the prediction of fiber orientation during processing [29,31,36,37].

Yet, most of the models that predict flow and rod orientation in fiber suspensions assume that the matrix is a Newtonian fluid. However, cement-based materials, even self-consolidating ones, exhibit most of the time plastic yield stress [38,39] and nonNewtonian phenomena, such as shear thinning and shear thickening behavior. In the range of shear rate applied in common concrete processes, concrete can be considered as a viscoplastic Bingham material which exhibits a linear viscous behavior when the yield stress is exceeded $[29,40]$.

Assuming a neglecting yield stress leads to discrepancy between modeling results and experimental observations. Viscous-based models predict a perfect alignment of the fiber in the flow direction while it is not experimentally the case because the material yield stress induces a constant resisting moment that cannot be overcome by the flow when a critical orientation is reached. It has recently been shown that the concrete or mortar shear yield stress can be predicted from both the cement paste yield stress and the volume fraction of immersed particles using micromechanical homogenization approaches [30,32,41-43].

Fiber suspensions can be characterized according to the volumetric fraction of solid particles in the fluid. Depending on the fiber volume fraction $\phi$, some quantities such as hydrodynamic or fiber-fiber interaction forces dominate the system. Typically, 
three distinct regimes are observed in the literature [44]. In the dilute regime, defined by $\phi<1 / r^{2}$ where $r$ is the aspect ratio, $L / D$ (length over diameter), the fibers are allowed to move without any interference from the other particles. By increasing the number of fibers, $1 / r^{2}<\phi<1 / r$, the semi-dilute domain is reached with non-negligible hydrodynamic interactions between fibers. Furthermore, some fiber contacts are also possible. Finally the concentrated regime is obtained when each fiber experiences numerous contacts with its neighbors: in this state, $\phi>1 / r$. Philipse $[45,46]$ suggested to describe the dense packing fraction of elongated particles $(r \gg 1)$ as $\phi_{f m}=\alpha_{m} / r$ and the loose packing fraction as $\phi_{f c}=\alpha_{c} /$ $r$. For random fiber orientation with an aspect ratio included between 50 and 100, Philipse [46] experimentally obtained $\alpha_{m}=4$ and $\alpha_{c}=3.2$, respectively. Therefore, Martinie [30] defined three regions with different degrees of fiber interaction. When $\phi$ $<\phi_{f c}$, the rheological behavior of the fiber suspension is close to the suspending fluid. Increasing the volume fraction until $\phi_{f c}<\phi$ $<\phi_{f m}$ induces that contact network between fibers influences the rheological behavior of the cementitious material. Finally, the material is not able to flow when $\phi>\phi_{f m}$.

In the literature, few theories deal with the fiber orientation in fiber-reinforced concrete or more generally in viscoplastic fluids, during their forming and most of them are based on the research field of molding of short fiber-reinforced polymers [36,47]. Therefore the governing equations written in the framework of suspensions of straight cylinders immersed in a Newtonian fluid applied to fiber-reinforced concretes, even though the cement paste behavior is not perfectly Newtonian.

Jeffery [48] developed a model for a single dilute spheroid suspended into a Newtonian fluid. The particle orientation is described by a unit vector $\mathbf{p}^{\alpha}$, directed along its principal axis, and its time evolution is given by the so-called Jeffery equation as:

$\dot{\mathbf{p}}_{J}^{\alpha}=-\frac{1}{2} \boldsymbol{\omega} \cdot \mathbf{p}^{\alpha}+\frac{\lambda}{2}\left(\dot{\gamma} \cdot \mathbf{p}^{\alpha}-\dot{\gamma}: \mathbf{p}^{\alpha} \mathbf{p}^{\alpha} \mathbf{p}^{\alpha}\right)$,

where $\omega$ and $\dot{\gamma}$ are the vorticity and deformation rate tensors, respectively. The shape factor $\lambda=\left(r^{2}-1\right) /\left(r^{2}+1\right)$ is a parameter related to the particle aspect ratio $r$. For a cylindrical particle, its length $L$ is greater than its diameter $D$ and the slender body theory [49-51] indicates that $\lambda=1$ (i.e. the thickness of the particle is neglected). The orientation state of a fiber population can be represented by a probability distribution function $\psi\left(\mathbf{p}^{\alpha}\right) \equiv \psi_{\mathbf{p}^{\alpha}}[52]$. Therefore, Advani and Tucker [52] defined its moments called orientation tensors, which compactly and efficiently characterize fiber orientation. The second- and fourth-order orientation tensors $\mathbf{a}_{2}$ and $\mathbf{a}_{\mathbf{4}}$ are given by:

$\mathbf{a}_{2}=\int_{\mathbf{p}^{\alpha}} \mathbf{p}^{\alpha} \mathbf{p}^{\alpha} \psi_{\mathbf{p}^{\alpha}} d \mathbf{p}^{\alpha}$,

$\mathbf{a}_{\mathbf{4}}=\int_{\mathbf{p}^{\alpha}} \mathbf{p}^{\alpha} \mathbf{p}^{\alpha} \mathbf{p}^{\alpha} \mathbf{p}^{\alpha} \psi_{\mathbf{p}^{\alpha}} d \mathbf{p}^{\alpha}$

The scalar measure of orientation for 3D orientation field can be defined by [53]:

$f=1-27 \operatorname{det}\left(\mathbf{a}_{2}\right)$.

$f$ varies from 0 in case of a random orientation to 1 in case of a perfectly aligned orientation.

The main difficulty in modeling the behavior of fiber-reinforced viscoplastic materials comes from the fact that these materials are multiscale and contain many interacting constituents. This study aims to develop an original constitutive law that describes the anisotropic plastic behavior of the material induced by the anisotropic orientation of fibers. This constitutive law provides new theoretical tools that enable to improve the modeling of fiber-reinforced materials exhibiting viscoplastic behaviors. This theory represents a first step toward a complete description of fiber-reinforced concrete rheology.

The overall objective of this work is divided into six distinct parts. The first one consists in describing the key steps in the derivation of the rheological model, in which interactions between fibers are taken into account. In the two following parts, the model is tested in simple shear flows and some analytical solutions are given for isotropic fiber orientation. The fourth part deals with the fiber orientation dynamics. The materials and the experimental methodology are detailed in the fifth part. Finally, the proposed model contains a few adjustable parameters that are determined by fitting experimental data (i.e. yield stress values) for steel fiber-filled reinforced kaolin pastes.

\section{Model formulation}

We supposed that fibers have a rod-like shape with a uniform length $L$ and a constant diameter $D$ and large aspect ratio $r \gg 1$. Furthermore, they are assumed rigid and are suspended in a viscoplastic fluid. This material consists of mono-disperse rigid non-colloidal and non-Brownian spherical particles (i.e. sand) suspended in a cement paste modeling as an incompressible yield stress fluid that follows a Herschel-Bulkley law. To separate the different constituent contributions, it is assumed that fiber length is greater than spherical inclusion diameter, which is larger than cement (or other binder) particle size. Therefore, the fiber-aggregate contacts are not taken into account. Then, the proposed constitutive law could be applied to any pasty suspension described by a Bingham or Herschel-Bulkley model and containing elongated rigid inclusions.

The total stress tensor $\boldsymbol{\sigma}$ for a suspension is made up of the extra stress tensor $\tau$ and a hydrostatic contribution due to the pressure $P$ :

$\boldsymbol{\sigma}=-P \boldsymbol{\delta}+\tau$,

where $\delta$ represents the identity tensor. The total extra stress tensor $\tau$ of the composite is:

$\tau=\tau^{c m}+\tau^{f}$

$\tau^{c m}$ and $\tau^{f}$ represent the deviatoric stress tensors for the cementitious material and fiber contributions, respectively.

\subsection{Extra stress of rigid spheres in Herschel-Bulkley fluids}

The cementitious material stress comes from the adding volume fraction $\phi_{s}$ of spherical particles in a Herschel-Bulkley fluid with a yield stress $\tau_{0}^{c m}$, a consistency $K$ and a power-law index $n$. Therefore, the cementitious material state equation is given by [41]:

$\left\{\begin{array}{ccc}\dot{\gamma}=\mathbf{0} & \text { if } & \tau^{c m} \leqslant \tau_{\phi_{s}}^{c m} \\ \tau^{c m}=\frac{\tau_{\phi_{s}}^{c m}}{\gamma} \dot{\gamma}+K_{\phi_{s}} \dot{\gamma}^{n-1} \dot{\gamma} & \text { if } & \tau^{c m} \geqslant \tau_{\phi_{s}}^{c m}\end{array}\right.$

where the effective yield stress is:

$\tau_{\phi_{s}}^{c m}=\tau_{0}^{c m} \sqrt{\left(1-\phi_{s}\right)\left(1-\phi_{s} / \phi_{m}\right)^{-2.5 \phi_{m}}}$

and the effective consistency reads as:

$K_{\phi_{s}}=K\left(1-\phi_{s}\right)^{(1-n) / 2}\left(1-\phi_{s} / \phi_{m}\right)^{-1.25(n+1) \phi_{m}}$.

$\phi_{m}$ refers to the maximum packing fraction. $\dot{\gamma}$ and $\tau^{\mathrm{cm}}$ are the magnitude of the deformation rate tensor $\dot{\gamma}$ and the suspending medium stress tensor $\tau^{c m}$, respectively. Note that the presence of spherical particles does not change the power-law index. It is also important to remark that no interactions between rigid spheres and fibers are 
taken into account. It means that the matrix is considered as a homogeneous material.

\subsection{Extra stress of rigid fiber suspended into a pseudoplastic fluid}

As fibers are considered as slender bodies, their thickness can be ignored and Brownian motion is neglected as particles are large enough. It is assumed that addition of fibers in a cementitious material contributes to its effective consistency through the hydrodynamic interactions and its effective yield stress due to the rodrod interactions, therefore $\tau^{f}=\tau_{H}^{f}+\tau_{I}^{f}$. The stress induced by fibers suspended into a pseudoplastic fluid is quite unrecognized [54,55]. The homogenization of the matrix contribution with a non-linear power-law is not straightforward, and it is assumed the same power-law as for the matrix (i.e. cementitious material). As a result, the contribution of the fibers to the hydrodynamic stress tensor is [55]:

$\tau_{H}^{f}=K_{\phi_{s}} \phi_{f} \frac{r^{n+1}}{2^{2 n-1}(n+2) \pi} \xi^{n} \mathbf{a}_{\mathbf{4}}^{(n)}: \dot{\gamma}$,

where $\mathbf{a}_{\mathbf{4}}^{(n)}$ is a fourth-order tensor called $n$-orientation tensor, dependant of the strain rate tensor as already proposed by Souloumiac and Vincent [54], and defined as:

$\mathbf{a}_{\mathbf{4}}^{(n)}=\int_{\mathbf{p}^{\alpha}} \mathbf{p}^{\alpha} \mathbf{p}^{\alpha} \mathbf{p}^{\alpha} \mathbf{p}^{\alpha}\left|\dot{\gamma}: \mathbf{p}^{\alpha} \mathbf{p}^{\alpha}\right|^{n-1} \psi_{\mathbf{p}^{\alpha}} d \mathbf{p}^{\alpha}$.

$K_{\phi_{s}}$ and $n$ are the effective homogeneous matrix consistency (i.e. the cement based matrix filled with spherical particles) and its powerlaw index, respectively. $\phi_{f}$ represents the fiber volume fraction and $\xi$ is the average parallel drag coefficient of the fiber. We note that Eq. (10) associated with Eq. (11) reduce to the typical constitutive equation for the stress of fibers suspended into a Newtonian matrix if for $n=1$ and $K_{\phi_{s}}=\eta_{0}[51,56]$ :

$\tau_{H}^{f}=\eta_{0} \phi_{f} \frac{r^{2}}{6 \pi} \xi \mathbf{a}_{\mathbf{4}}: \dot{\gamma}$.

Eq. (10) or Eq. (12) describe the coupling between hydrodynamic forces and fiber orientation and hence $\xi$ is also called the coupling coefficient. These both expressions are valid for semi-concentrated systems and even in these regimes, fiber-fiber interactions are encountered. Therefore, an additional stress contribution due to the contact forces acting between fibers must be introduced [56]. The interaction force is assumed to be a lubrication force with a non-linear power-law form [57]. Natale et al. [57] showed that their model predictions are in good agreement with the experimental findings for both suspensions of glass fibers in a Newtonian polybutene and carbon nanotubes in a Newtonian epoxy resin, demonstrating its ability to describe the behavior of micro- and nanoscale rod suspensions. For a power-law index between 0 and 1, Natale et al. [57] found that particle interactions become predominant at low-shear rates, exhibiting an apparent yield stress. To predict a pure yield stress behavior, the powerlaw exponent is set to zero, indicating that the interaction force is proportional to the relative velocity at the contact point and to the scalar $\left|\dot{\gamma}: \mathbf{p}^{\alpha} \mathbf{p}^{\alpha}\right|^{-1}$ (some details are given below). Therefore, the contribution to the extra stress due to fiber interaction becomes:

$\tau_{I}^{f}=b \phi_{f}^{2} \frac{8 r^{2}}{\pi^{2} L} k \mathbf{b}_{4}^{(0)}: \dot{\gamma}$,

where $b$ is the consistency and $k$ is a parameter related to the intensity of the rod-rod interaction force. In the following, the superscript $\alpha$ refers to the test fiber while $\beta$ is used for the neighboring fiber. $\mathbf{b}_{\mathbf{4}}^{(0)}$ is a fourth-order tensor called the $n$-interaction tensor and has already been proposed by Natale et al. [57]. Its definition is given by:

$\mathbf{b}_{\mathbf{4}}^{(0)}=\int_{\mathbf{p}^{\alpha}} \int_{\mathbf{p}^{\beta}} \mathbf{p}^{\alpha} \mathbf{p}^{\alpha} \mathbf{p}^{\alpha} \mathbf{p}^{\alpha}\left|\mathbf{p}^{\alpha} \times \mathbf{p}^{\beta} \| \dot{\gamma}: \mathbf{p}^{\alpha} \mathbf{p}^{\alpha}\right|^{-1} \psi_{\mathbf{p}}^{\alpha} \psi_{\mathbf{p}}^{\beta} d \mathbf{p}^{\beta} d \mathbf{p}^{\alpha}$.

This tensor is completely symmetric and shows a direct dependence on the rate of deformation. It is defined by forming the dyadic products of the unit vector $\mathbf{p}^{\alpha}$, weighted by the Onsager potential $\left|\mathbf{p}^{\alpha} \times \mathbf{p}^{\beta}\right|$ and the scalar $\left|\dot{\gamma}: \mathbf{p}^{\alpha} \mathbf{p}^{\alpha}\right|^{-1}$, and then twice integrating the product with respect to the distribution function over all possible directions. The Onsager potential is directly proportional to the contact probability that represents the probability that a neighboring fiber interacts with the test fiber. Its maximum equals one when two fibers interact orthogonally and its minimum is null when two rods are both parallel [58]. $\psi_{\mathbf{p}^{\beta}}$ is the probability to find an interacting fiber $\beta$ with an orientation $\mathbf{p}^{\beta}$. The scalar quantity $\left|\dot{\gamma}: \mathbf{p}^{\alpha} \mathbf{p}^{\alpha}\right|^{-1}$ is related to the non-linear intensity of the interaction force.

\subsection{Summary of the model}

In summary, the fiber-reinforced viscoplastic fluids state equation reads:

$\boldsymbol{\sigma}=-P \boldsymbol{\delta}+\boldsymbol{\tau}$,

$\left\{\begin{array}{ccc}\dot{\gamma}=\mathbf{0} & \text { if } & \tau \leqslant \tau_{Y} \\ \tau=\tau_{Y}+\tau_{V} & \text { if } & \tau \geqslant \tau_{Y}\end{array}\right.$,

where the yield stress contribution arises from the pasty suspension and the fiber-fiber interactions, such as:

$\tau_{Y}=\frac{\tau_{\phi_{s}}^{\mathrm{cm}}}{\dot{\gamma}} \dot{\gamma}+b \phi_{f}^{2} \frac{8 r^{2}}{\pi^{2} L} k \mathbf{b}_{4}^{(0)}: \dot{\gamma}$

The viscous stress contribution is given by the sum of the pasty suspension consistency and the fiber hydrodynamic interactions:

$\tau_{V}=K_{\phi_{s}} \dot{\gamma}^{n-1} \dot{\gamma}+K_{\phi_{s}} \phi_{f} \frac{r^{n+1}}{2^{2 n-1}(n+2) \pi} \xi^{n} \mathbf{a}_{4}^{(n)}: \dot{\gamma}$.

$\tau$ and $\tau_{Y}$ are the second invariants defined by $\tau=\sqrt{\tau: \tau / 2}$ and $\tau_{Y}=\sqrt{\tau_{Y}: \tau_{Y} / 2}$, respectively. It is important to note that the last term in Eq. (18) involves $\mathbf{a}_{\mathbf{4}}^{(n)}$ which is directly related to the fiber orientation and to the magnitude of the deformation rate tensor. All the above expressions are derived for a suspending material that follows the Herschel-Bulkley law. If $n=1$ and $K=\eta_{0}$, these expressions can be used to describe the behavior of a fiber-reinforced mortar, where the suspending medium follows a Bingham law.

For the case of steady-state shear flow, the viscosity is deduced from Eq. (16) and can be written as:

$$
\left\{\begin{array}{cc}
\eta=\infty & \text { if } \tau \leqslant \tau_{Y} \\
\eta=\frac{\tau_{\phi_{s}}^{c m}}{\dot{\gamma}}+2 \phi_{f}^{2} r^{2} B b_{1122}^{(0)}+K_{\phi_{s}} \dot{\gamma}^{n-1}+2 K_{\phi_{s}} \phi_{f} r^{n+1} A a_{1122}^{(n)} & \text { if } \tau \geqslant \tau_{Y}
\end{array},\right.
$$

where $A=\xi^{n} /\left[2^{2 n-1}(n+2) \pi\right]$ and $B=8 k b /\left(\pi^{2} L\right)$, respectively. The first two terms of the right hand side of Eq. (19) results from the yield stress of the composite and the last two terms are the viscous contribution. Eq. (19) also shows that some key components of the fourth-order tensors (i.e. $\mathbf{a}_{\mathbf{4}}^{(n)}$ and $\mathbf{b}_{4}^{(0)}$ ) are needed to evaluate the viscosity. Closure approximations can be developed to express the fourth-order tensors in terms of the second-order ones based on the following works [59-61]. Even though closure approximations are more and more accurate, they lead to some unavoidable errors. Another possible strategy is to numerically solve the associated Fokker-Planck equation by using numerical approaches such as proper generalized decomposition [62,63]. Once, the distribution 


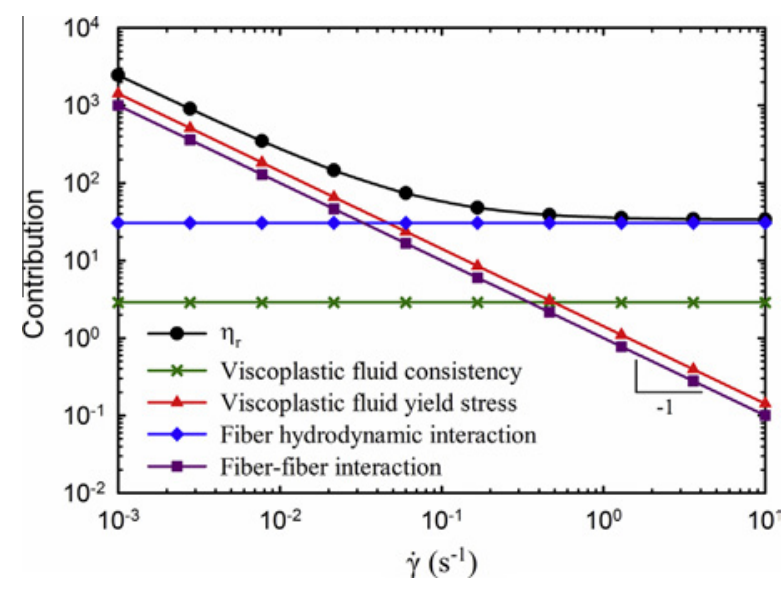

Fig. 1. Individual and overall contributions to the steady-state reduced viscosity of a fiber-reinforced viscoplastic fluid in simple shear flow. The results are obtained in the case of isotropic fiber orientation distribution and $n=1$.

function is numerically computed and then the $a_{1122}^{(n)}$ and $b_{1122}^{(0)}$ components are straightforwardly obtained for any shear rate using Eqs. (11) and (14) without any approximations [64].

\section{Isotropic orientation distribution in simple shear flows}

In order to understand what the model predicts, a first set of simulations was computed imposing an isotropic orientation distribution. This condition was chosen because it is the orientation state generally assumed after a mixing. All the predictions presented in this section are obtained for the following conditions: $K=50 \mathrm{~Pa} \mathrm{~s}^{n}, \tau_{0}^{c m}=50 \mathrm{~Pa}, \phi_{s}=30 \%, \phi_{m}=58 \%, \phi_{f}=5 \%, r=40, A=1$ and $B=75 \mathrm{~Pa}$. These values are those generally encountered with rigid fiber-reinforced cement-based materials [32] except for the two last ones, which are chosen to highlight the model predictions. It is interesting to note that the parameters considered in this first set of simulations correspond to a semi-concentrated regime. In the case of simple shear flow and for $n=1$ (it means that the suspending medium is a Bingham fluid), the four contributions of Eq. (19) to the steady-state reduced viscosity of a fiber-reinforced cementitious material are illustrated in Fig. 1. The hydrodynamic contribution of the viscoplastic fluid is enhanced by the presence of the spherical particles and is independent of the shear rate. The same result is observed for the rod hydrodynamic contribution. At low shear rates, the yield stress exhibited by the viscoplastic fluid and the fiber-fiber interactions are the dominant mechanics while, at high shear rates, the hydrodynamic contributions become controlling. The transition between the two mechanics is clearly represented by the trend of the reduced viscosity that goes from a solid-like behavior to a plateau-like at high shear rates that reaches the viscosity of the fiber suspension.

The effect of the power-law index $n$ on the reduced viscosity is highlighted in Fig. 2 for shear rates up to $100 \mathrm{~s}^{-1}$, which is not really realistic. Varying $n$ from 0.8 to 1.2 , a full spectrum of behavior is obtained, from shear thinning $(n<1)$ to shear thickening $(n>1)$ and include Newtonian $(n=1)$. Hence, the power-law index $n$ controls the behavior of the suspensions at high shear rates. At low shear rates, solid-like behaviors are observed independently of the values of $n$.

\section{Analytical solution for an isotropic orientation state in simple shear flows}

The aim of this part is to provide some analytical tools to easily express some components of the fourth-order tensors in simple shear with an isotropic fiber orientation. Numerical solutions

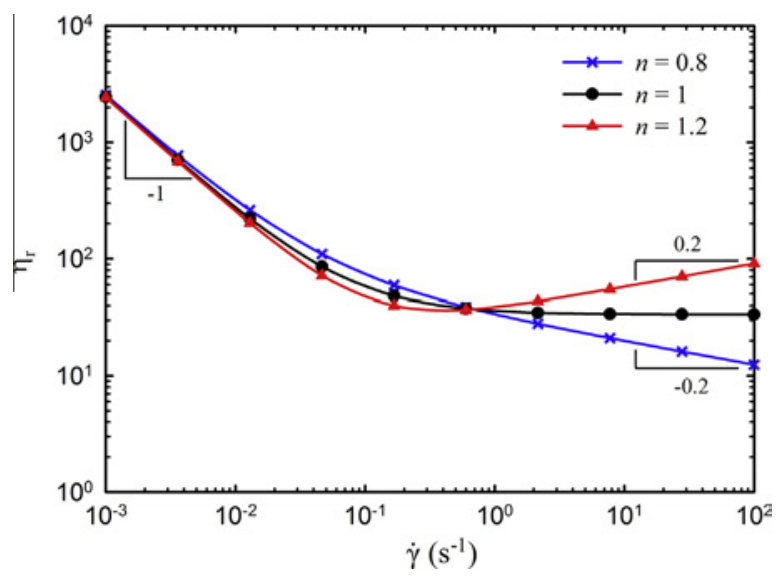

Fig. 2. Reduced steady shear viscosity predictions as function of shear rate for different values of the power-law index $n$.

obtained by the discretization of probability distribution functions are not very practical to evaluate the key components of the fourth-order tensors $\mathbf{a}_{\mathbf{4}}^{(n)}$ and $\mathbf{b}_{\mathbf{4}}^{(0)}$. Therefore we attempted to express some analytical solutions in simple shear flows considering an isotropic fiber orientation. In that case, $a_{1122}^{(n)}$ needs to be related to the applied shear rate $\dot{\gamma}$ and to the power-law index $n$. When $n=1$, a linear closure relation for the fourth-order orientation tensor [52] gives $a_{1122}^{(n=1)}=1 / 15$, which is the exact value for isotropic orientation and holds for any values of shear rates. In the limit where $n=0$, it is possible to perform some analytical integrations of Eq. (11) and it results that $a_{1122}^{(n=0)}=1 / 3 \pi|\dot{\gamma}|$ (see Appendix A for calculation details). Fig. 3 depicts the $a_{1122}^{(n=1)}$ and $a_{1122}^{(n=0)}$ components for simple shear flow. It can be observed that these two curves intersect at a critical shear rate of $\dot{\gamma}_{c}=5 / \pi \mathrm{s}^{-1}$. At $\dot{\gamma}_{c}$, the average force exerted by a power-law fluid on fibers having an isotropic orientation is equivalent to the average force exerted by a Newtonian matrix for the same fiber orientation state. Therefore it can be deduced that the $a_{1122}^{(n)}$ component for on isotropic orientation state writes as:

$a_{1122}^{(n)}=\frac{1}{15}\left|\frac{\pi}{5} \dot{\gamma}\right|^{n-1}$.

For the cases of $n=0.3$ (shear thinning behavior) and $n=1.5$ (shear thickening behavior), Eq. (20) is compared to the numerical integration of Eq. (11) and the obtained results are indistinguishable. Up to now, no obvious analytical solutions are available for

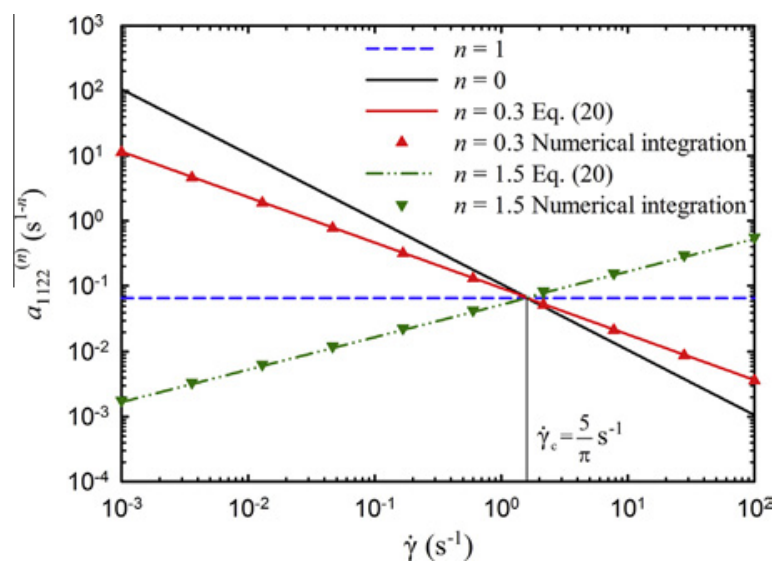

Fig. 3. Variations of the $a_{1122}^{(n=1)}$ and $a_{1122}^{(n=0)}$ components as a functions of shear rate in for simple shear flow. Also shown are the results obtained with Eq. (20) and numerical integration for the case of $n=0.3$ and $n=1.5$. 


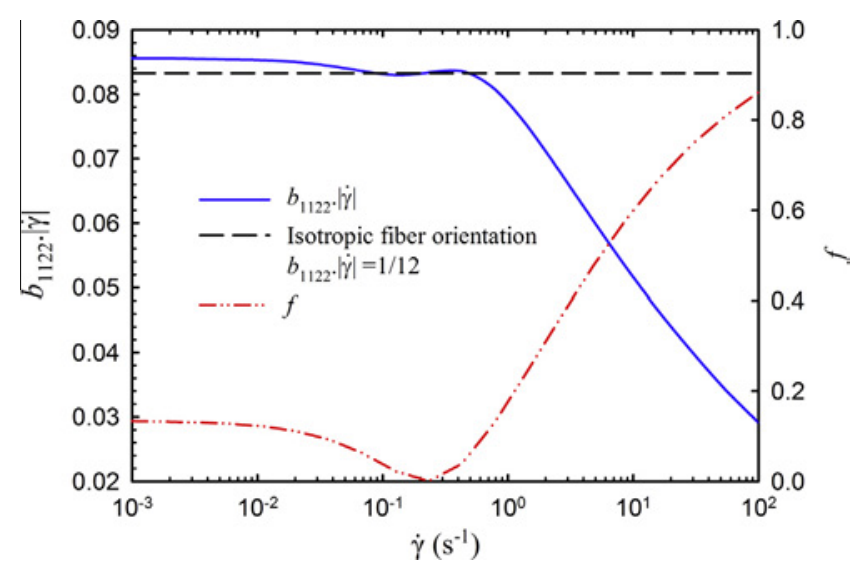

Fig. 4. Variations of the $b_{1122}^{(0)}$ component normalized with the shear rate as functions of shear rate. The black long dash line indicates the normalized $b_{1122}^{(0)}$ component value for an isotropic orientation state. Also shown is the variation of the scalar measure of orientation $f$.

fiber orientation states different from isotropic (except when fibers are perfectly aligned in one direction) and for arbitrary flow conditions.

It remains to find a way to express $b_{1122}^{(0)}$. Eq. (14) involves the Onsager potential $\left|\mathbf{p}^{\alpha} \times \mathbf{p}^{\beta}\right|$ that is often replaced by the MaierSaupe potential $1-\mathbf{p}^{\alpha} \mathbf{p}^{\alpha}: \mathbf{p}^{\beta} \mathbf{p}^{\beta}$ corrected by numerical prefactors to reach the exact value for the isotropic orientation state $[56,65]$. Thus straightforward algebraic calculations yield to (see Appendix B):

$b_{1122}^{(0)}=\frac{1}{12|\dot{\gamma}|}$.

Note that the difference between the analytical solution [Eq. (21)] and the numerical integration of Eq. (14) is around $1.2 \%$, especially at low shear rates (a $120 \times 120$ cell grid was used [64]).

Thanks to these analytical solutions for an isotropic fiber orientation, the viscosity in the case of steady-state shear flow becomes:

$$
\left\{\begin{array}{ccc}
\eta=\infty & \text { if } \tau \leqslant \tau_{Y} \\
\eta=\frac{\tau_{\phi_{s}}^{c m}}{\dot{\gamma}}+\frac{1}{6} \frac{\phi_{f}^{2} r^{2} B}{\dot{\gamma}}+K_{\phi_{s}} \dot{\gamma}^{n-1}+\frac{2}{15} K_{\phi_{s}} \phi_{f} r^{n+1} A\left(\frac{\pi}{5} \dot{\gamma}\right)^{n-1} & \text { if } \tau \geqslant \tau_{Y}
\end{array} .\right.
$$

The absolute value has been removed as positive applied shear rate is assumed. Finally, with the same set of constitutive parameters used in part 3 , the model predictions give identical results of those presented in Fig. 2 (results not shown).

\section{Fiber orientation dynamics}

Natale et al. [57] showed that the expression of the orientation time evolution for the test fiber $\alpha$ is given by:

$$
\begin{aligned}
\dot{\mathbf{p}}^{\alpha}= & \dot{\mathbf{p}}_{J}^{\alpha} \\
& -\phi_{f} N_{I}\left(\frac{1}{2} \dot{\gamma} \cdot \mathbf{p}^{\alpha}-\frac{1}{2} \dot{\gamma}: \mathbf{p}^{\alpha} \mathbf{p}^{\alpha} \mathbf{p}^{\alpha}+q|\dot{\gamma}| \frac{\partial \ln \psi_{\mathbf{p}^{\alpha}}}{\partial \mathbf{p}^{\alpha}}\right)\left|\dot{\gamma}: \mathbf{p}^{\alpha} \mathbf{p}^{\alpha}\right|^{-1} \\
& \times \int\left|\mathbf{p}^{\alpha} \times \mathbf{p}^{\beta}\right| \psi_{\mathbf{p}^{\beta}} d \mathbf{p}^{\beta},
\end{aligned}
$$

where $\dot{\mathbf{p}}_{J}^{\alpha}$ is the Jeffery equation [Eq. (1)] and $q$ is a dimensionless interaction coefficient. This diffusional term (the third term in the brackets in Eq. (23)) is not due to the Brownian motion, but represents the rotational fluctuations undergoing by the test fiber, when the suspension is under flow. Finally, $N_{\mathrm{I}}$ is directly proportional to the intensity of the friction between particles.

From Bird et al. [66], the probability distribution function $\psi_{\mathbf{p}^{\alpha}}$ may be regarded as a convected quantity:

$\frac{D \psi_{\mathbf{p}^{\alpha}}}{D t}=-\frac{\partial}{\partial \mathbf{p}^{\alpha}} \cdot\left(\dot{\mathbf{p}}^{\alpha} \psi_{\mathbf{p}^{\alpha}}\right)$,

where $D / D t$ represents the material derivative and reduces to partial derivative in the case of homogenous flows. Combining the previous conservation equation [Eq. (24)] and the fiber angular velocity equation [Eq. (23)] leads to the governing equation known as the Fokker-Planck equation. The probability distribution function evaluated for steady-state is:

$$
\begin{aligned}
\frac{\partial}{\partial \mathbf{p}^{\alpha}} \cdot\left(\dot{\mathbf{p}}_{I}^{\alpha} \psi_{\mathbf{p}^{\alpha}}\right)= & \phi_{f} N_{I} q|\dot{\gamma}| \frac{\partial}{\partial \mathbf{p}^{\alpha}} \\
& \cdot\left(\frac{\partial \psi_{\mathbf{p}^{\alpha}}}{\partial \mathbf{p}^{\alpha}}\left|\dot{\gamma}: \mathbf{p}^{\alpha} \mathbf{p}^{\alpha}\right|^{-1} \int\left|\mathbf{p}^{\alpha} \times \mathbf{p}^{\beta}\right| \psi_{\mathbf{p}}^{\beta} d \mathbf{p}^{\beta}\right),
\end{aligned}
$$

where

$$
\begin{aligned}
\dot{\mathbf{p}}_{I}^{\alpha}= & \dot{\mathbf{p}}_{J}^{\alpha}-\frac{1}{2} \phi_{f} N_{I}\left(\dot{\gamma} \cdot \mathbf{p}^{\alpha}-\dot{\gamma}: \mathbf{p}^{\alpha} \mathbf{p}^{\alpha} \mathbf{p}^{\alpha}\right) \mid \dot{\gamma} \\
& :\left.\mathbf{p}^{\alpha} \mathbf{p}^{\alpha}\right|^{-1} \int\left|\mathbf{p}^{\alpha} \times \mathbf{p}^{\beta}\right| \psi_{\mathbf{p}^{\beta}} d \mathbf{p}^{\beta} .
\end{aligned}
$$

Solving the orientation fields via the probability distribution is detailed in [57] and [64]. Therefore, it is possible to obtain microstructural information from the model predictions. More precisely, the steady-state components of $b_{1122}^{(0)}$ are numerically computed in simple shear flow. The parameter values used for the calculation are $\phi_{\mathrm{f}} N_{\mathrm{I}}=0.1 \mathrm{~s}^{-1}$ and $q=0.25$. The evolution of $b_{1122}^{(0)}|\dot{\gamma}|$ versus $\dot{\gamma}$ is plotted in Fig. 4. For the suspension, the scalar measure of orientation $f$ [Eq. (4)] can be used as a compact representation of the overall orientation of the fibers without significant loss of information and is also illustrated in Fig. 4. Initially, the fibers tend toward an isotropic orientation until a shear rate around $1 \mathrm{~s}^{-1}$, and only at larger shear rates do they orient along the flow direction. This is confirmed by the scalar $f$ which depicts a steady value close to 0.2 , indicating a quasi-isotropic fiber orientation, and then increases until 1, corresponding to a fully orientation in the flow direction. A small undershoot is observed at a shear rate around $0.2 \mathrm{~s}^{-1}$. Until the shear rate at which the minimum appears, the tendency of the fibers to get oriented in the flow direction is overcome by the particle-particle interactions. At higher shear rates the hydrodynamic interactions become more important and the fibers get oriented. The $b_{1122}^{(0)}$ component for the isotropic orientation state is also given in Fig. 4 for information purposes.

Fig. 4 is also useful to express the yield stress value for different fiber orientation states. From Eq. (19), the yield stress of the composite becomes:

$\tau_{Y}=\tau_{\phi_{s}}^{c m}+2 \phi_{f}^{2} r^{2} B b_{1122}^{(0)} \dot{\gamma}$.

Therefore, the presence of fibers induces an anisotropic yield stress depending on how the fibers are oriented. Investigations in elongational flows were not performed.

\section{Materials and experimental methodology}

A kaolin paste was chosen to emphasize the rheological features due to the fibers in the suspension. The kaolin clay used was a Powdered Polwhite BB from Imerys ${ }^{\circledR}$ (Kaolins de Bretagne, Ploemeur, France). The specific gravity of the clay was 2.65 , the largest clay grain size approximately $40 \mu \mathrm{m}$ and mean grain size close to $9 \mu \mathrm{m}$. The kaolin powder specific area is $105 \mathrm{~cm}^{2} / \mathrm{g}$ and the water/kaolin mass ratio used is 1.1 . 
Steel fibers were dispersed in kaolin pastes in order to measure their yield stresses. Two cylindrical shapes with a constant diameter of $160 \mu \mathrm{m}$ are tested: their lengths are 6 and $13 \mathrm{~mm}$, leading to aspect ratios $r$ of 37.5 and 81, respectively. Their specific gravity is 7.85 and their Young modulus is $210 \mathrm{GPa}$. Note that the largest clay grain size, which represents $0.1 \%$ of clay grains, is of the same order as the fiber diameter ( $40 \mu \mathrm{m}$ as compared to $160 \mu \mathrm{m}$ ).

Six different weight fractions of fibers with $r=37.5$ were used corresponding to fiber factors, $r \phi_{f}$ [67] of $0.75,1.5,2.25,3,3.38$ and 3.56, respectively, and four different weight fractions of fibers with $r=81$ were used corresponding to fiber factors of $0.81,1.62$, 2.43 and 3.24, respectively. Therefore, all the investigated fiber suspensions range from the dilute to the concentrated regimes.

Kaolin and water were firstly mixed in a Hobart mixer. Then, the material was poured in five different cylindrical containers of $10 \mathrm{~cm}$ in diameter and $15 \mathrm{~cm}$ in height. Fibers were manually added and dispersed in the paste in order to obtain a random homogeneous dispersion. This procedure also ensures us that the fiber orientation is close to the isotropic state.

An Anton Paar Rheolab QC rheometer equipped with two vane geometries well adapted for mineral paste and mortar is used for the measurements. The geometries consist of four blades around a cylindrical shaft. The vane sizes are $60 \mathrm{~mm}$ in height and $40 \mathrm{~mm}$ in diameter for the first one, and $40 \mathrm{~mm}$ and $22 \mathrm{~mm}$, respectively, for the second one. Tests were carried out in an "infinite" gap in order to avoid wall effects.

A measurement stage was performed during $180 \mathrm{~s}$ on the rheometer to obtain the yield stress at five given resting times, following the procedure described by Mahaut et al. [43]. Stress growth is used to determine the yield stress with an apparent shear rate of $10^{-2} \mathrm{~s}^{-1}$. At such shear rate, viscosity effects are negligible. As a consequence, the yield stress is computed from the maximum torque $T$ value which is required for the onset of the flow, i.e. when the apparent yield stress (static yield stress) is reached on the cylindrical shearing surface:

$\tau_{0}=\frac{T}{\frac{\pi D^{2}}{2}\left(H+\frac{D}{3}\right)}$,

where $T$ is the torque peak value, $H$ is the tool height and $D$ is its diameter. The measured yield stress values of the unfilled kaolin pastes are found to be $404 \pm 32 \mathrm{~Pa}$ for the suspensions with $r=37.5$ and $521 \pm 14 \mathrm{~Pa}$ for the suspensions with $r=81$, respectively.

\section{Data and predictions}

Fig. 5 depicts the relative yield stress $\tau_{Y} / \tau_{\phi_{s}}^{c m}$ (i.e. ratio between the yield stress of the kaolin paste containing fibers and the yield stress of the kaolin paste without any fibers) as a function of the fiber factor, $r \phi_{f}$. Due to the operating method, the fiber orientation state for the different suspensions is assumed isotropic. With the addition of the fibers, the relative yield stress increases with a significant enhancement at fiber factor close to 3.5. This effect is more pronounced as the fiber aspect ratio equals 37.5. The relative yield stress data with $r=81$ describe linear dependences at low fiber factors and then exhibit quadratic trends as fiber contents increase.

The predicted relative yield stress for an isotropic fiber orientation state is obtained from Eq. (27) using Eq. (21):

$\frac{\tau_{Y}}{\tau_{\phi_{s}}^{c m}}=1+\frac{\phi_{f}^{2} r^{2} B}{6 \tau_{\phi_{s}}^{c m}}$.

Results are given in Fig. 5 by the dash lines using the parameters $B=1200 \mathrm{~Pa}$ for $r=37.5$ and $B=2924 \mathrm{~Pa}$ for $r=81$. The fits are in very good agreement with the experimental data. However, some discrepancies are observed for suspensions with fibers having an

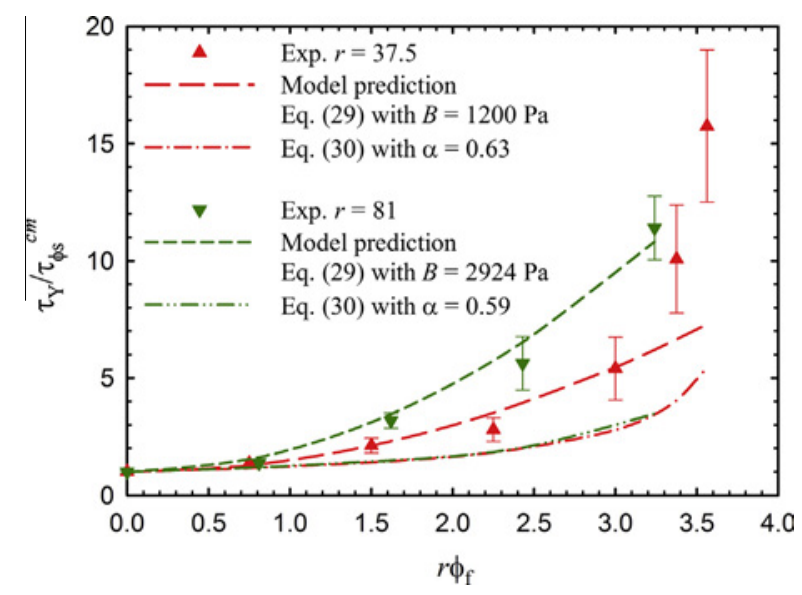

Fig. 5. Relative yield stress as a function of the fiber factor. Results for the model predictions are also shown.

aspect ratio of $r=37.5$ and a fiber factor larger than 3 . It may be explained by the validity the fiber contact probability, which was derived for large aspect ratio (i.e. $r \gg 1$ ) [56,68].

It is interesting to do an analogy between fibers having an isotropic orientation and inclusions with spherical shape for the prediction of the yield stress. Martinie et al. [30] have defined an equivalent fiber solid volume fraction $\phi_{f, e q}=\phi_{s m} \phi_{f} r / 4$ for reinforced suspensions. This equivalent fiber solid volume fraction replaces the spherical particle fraction in the yield stress prediction of Chateau et al. [41]. Based on the work of Perrot et al. [32], it is then possible to determine the yield stress values for rigid fiberreinforced suspensions as:

$\frac{\tau_{Y}}{\tau_{\phi_{s}}^{c m}}=\sqrt{\frac{1-\phi_{s m} \phi_{f} \frac{r}{4 \alpha}}{\left(1-\phi_{f} \frac{r}{4}\right)^{2.5 \frac{\phi_{s m}}{\alpha}}}}$

where the dense packing fraction is $\phi_{s m}=0.6$ [32]. The constant $\alpha$ (with $\alpha<1$ ) is defined as a fitting parameter, which is used to exactly match the fiber volume fraction corresponding to the yield stress divergence. The value of $\alpha$ is expected to be close to 1 in order to find a yield stress divergence for volume fraction ranging between $r / 3.2$ and $r / 4$, which are some critical fiber packing volume fractions defined in [46]. Fig. 5 reports the predicted relative yield stress given by Eq. (30). Results show that the two curves are indistinguishable, meaning that Eq. (30) is independent of the fiber aspect ratio for in the investigated range. Furthermore, the fittings obtained with $\alpha=0.63$ and $\alpha=0.59$ for $r=37.5$ and $r=81$, respectively, underestimate the experimental data for fiber factor higher than 1.

Our first observations show that the physics from which the model is derived is of a great relevance. The proposed assumptions seem to be quite reasonable but further efforts are necessary, mainly concerning the determination of the fiber contact probability. Indeed, the derivation of fiber contact probability corresponds to rods with their centers outside of an imaginary tube with a diameter of $2 D$ entering through the curved surface [69]. Improvements can be performed by considering fibers with their centers outside the tube, entering through the end surfaces [70] and with fibers having their centers within the tube [71]. In simple shear flows, exact solutions for isotropic fiber orientations are obtained but approximations should be proposed for other fiber orientation states. Improvement of the present work can also be accomplished by considering a contribution to the yield stress due to the presence of the fibers and not only to their orientation. Fig. 4 reports that $b_{1122}^{(0)}$ will be zero when fibers will be perfectly aligned, 
indicating that the yield stress of the suspension equals the one of the matrix. This is a paradox that should be corrected by including a finite thickness for the particles in the model.

\section{Conclusions}

A new constitutive law has been proposed to describe the yield stress and consistency of non-dilute fiber suspensions in viscoplastic materials. The total extra stress of the composite is considered as the sum of the contributions of the viscoplastic fluid (HerschelBulkley fluid filled with spherical inclusions) and fiber contributions (hydrodynamic and non-linear fiber-fiber interactions), respectively. This model is able to predict shear thinning and shear thickening behaviors as well as anisotropic yield stress depending on the fiber orientation. The extra stress term involves two fourthorder tensors, the orientation and interaction tensors, respectively, where both quantities are function of the effective deformation rate. In simple shear flow, analytical expressions for both tensors are given for an isotropic fiber orientation state.

In order to illustrate the model, a rheological study of suspensions with different fiber volume fractions have been performed using kaolin pastes and steel fibers having two distinct aspect ratios. The investigated fiber suspensions range from the dilute to the concentrated regimes. Results showed a fairly good agreement in terms of the predicted yield stress values. For kaolin pastes having the largest aspect ratio, the measured relative yield stress was found to be quadratic functions of the fiber factor, as predicted by the model. Only one parameter is required to predict the yield stress values for an isotropic fiber orientation. In this work, the expression for the anisotropic yield stress has not been experimentally validated.

\section{Appendix A}

In simple shear flow (where $\dot{\gamma}$ is the applied shear rate) and with $n=0$, the scalar quantity $\left|\dot{\gamma}: \mathbf{p}^{\alpha} \mathbf{p}^{\alpha}\right|^{n-1}$ involved in Eq. (11) writes:

$\left|\dot{\gamma}: \mathbf{p}^{\alpha} \mathbf{p}^{\alpha}\right|^{-1}=\left(2|\dot{\gamma} \cos (\phi) \sin (\phi)| \sin ^{2}(\theta)\right)^{-1}$,

where the components of the unit vector $\mathbf{p}^{\alpha}$, which gives the orientation of a single fiber, are related to the angles $(\theta, \phi)$ in spherical coordinates by [52]:

$p_{1}=\sin (\theta) \cos (\phi)$

$p_{2}=\sin (\theta) \sin (\phi)$.

$p_{3}=\cos (\theta)$

Therefore the $a_{1122}$ component of $\mathbf{a}_{\mathbf{4}}^{(0)}$ becomes for an isotropic fiber orientation (i.e. $\psi_{\mathbf{p}^{\alpha}}=1 / 4 \pi$ ):

$a_{1122}^{(n=0)}=\frac{1}{4 \pi} \int_{\phi=0}^{\phi=2 \pi} \int_{\theta=0}^{\theta=\pi} \frac{\cos ^{2}(\phi) \sin ^{2}(\phi) \sin ^{3}(\theta)}{2|\dot{\gamma} \cos (\phi) \sin (\phi)|} d \theta d \phi$

By performing some straightforward algebraic calculations, we obtain:

$a_{1122}^{(n=0)}=\frac{1}{3 \pi|\dot{\gamma}|}$.

\section{Appendix B}

The Onsager potential $\left|\mathbf{p}^{\alpha} \times \mathbf{p}^{\beta}\right|$, contained in the interaction tensor defined by Eq. (14), is generally approximated using Taylor series expansion until the first order. Then a prefactor is introduced to reach the exact value for the isotropic orientation state and it leads to [65]:

$\left|\mathbf{p}^{\alpha} \times \mathbf{p}^{\beta}\right| \approx \frac{5 \pi}{16}\left(1-\frac{3}{5} \mathbf{p}^{\alpha} \mathbf{p}^{\alpha}: \mathbf{p}^{\beta} \mathbf{p}^{\beta}\right)$.

Therefore, according to its definition Eq. (14) and using Eq. (35), $\mathbf{b}_{4}^{(n=0)}$ becomes for an isotropic fiber orientation (i.e. $\left.\psi_{\mathbf{p}^{\alpha}}=\psi_{\mathbf{p}^{\beta}}=1 / 4 \pi\right)$ :

$\mathbf{b}_{\mathbf{4}}^{(0)}=\frac{1}{16 \pi^{2}} \int_{\mathbf{p}^{\alpha}} \int_{\mathbf{p}^{\beta}} \mathbf{p}^{\alpha} \mathbf{p}^{\alpha} \mathbf{p}^{\alpha} \mathbf{p}^{\alpha} \frac{\pi}{8}\left[1+\frac{3}{2}\left(1-\mathbf{p}^{\alpha} \mathbf{p}^{\alpha}: \mathbf{p}^{\beta} \mathbf{p}^{\beta}\right)\right]\left|\dot{\gamma}: \mathbf{p}^{\alpha} \mathbf{p}^{\alpha}\right|^{-1} d \mathbf{p}^{\beta} d \mathbf{p}^{\alpha}$

In simple shear flow, calculations of the 1122-component of give:

$\frac{1}{16 \pi^{2}} \int_{\mathbf{p}^{\alpha}} \int_{\mathbf{p}^{\beta}} \mathbf{p}^{\alpha} \mathbf{p}^{\alpha} \mathbf{p}^{\alpha} \mathbf{p}^{\alpha}\left(1-\mathbf{p}^{\alpha} \mathbf{p}^{\alpha}: \mathbf{p}^{\beta} \mathbf{p}^{\beta}\right)\left|\dot{\gamma}: \mathbf{p}^{\alpha} \mathbf{p}^{\alpha}\right|^{-1} d \mathbf{p}^{\beta} d \mathbf{p}^{\alpha}=\frac{2}{9 \pi|\dot{\gamma}|}$,

and

$\frac{1}{16 \pi^{2}} \int_{\mathbf{p}^{\alpha}} \int_{\mathbf{p}^{\beta}} \mathbf{p}^{\alpha} \mathbf{p}^{\alpha} \mathbf{p}^{\alpha} \mathbf{p}^{\alpha}\left|\dot{\gamma}: \mathbf{p}^{\alpha} \mathbf{p}^{\alpha}\right|^{-1} d \mathbf{p}^{\beta} d \mathbf{p}^{\alpha}=\frac{1}{3 \pi|\dot{\gamma}|}$.

As a result, the $b_{1122}^{(0)}$ component of $\mathbf{b}_{4}^{(0)}$ writes as:

$b_{1122}^{(0)}=\frac{\pi}{8}\left(\frac{1}{3 \pi|\dot{\gamma}|}+\frac{3}{2} \frac{2}{9 \pi|\dot{\gamma}|}\right)=\frac{1}{12|\dot{\gamma}|}$

\section{References}

[1] S. Foster, The application of steel-fibres as concrete reinforcement in Australia: from material to structure, Mater. Struct. 42 (2009) 1209-1220.

[2] M. Lepech, V. Li, Application of ECC for bridge deck link slabs, Mater. Struct. 42 (2009) 1185-1195.

[3] P. Serna, S. Arango, T. Ribeiro, A.M. Nuez, E. Garcia-Taengua, Structural cast-inplace SFRC: technology, control criteria and recent applications in spain, Mater. Struct. 42 (2009) 1233-1246.

[4] P. Pujadas, A. Blanco, A. Fuente, A. Aguado, Cracking behavior of FRC slabs with traditional reinforcement, Mater. Struct. 45 (2012) 707-725.

[5] L. Ferrara, A. Meda, Relationships between fibre distribution, workability and the mechanical properties of SFRC applied to precast roof elements, Mater. Struct. 39 (2006) 411-420.

[6] M. di Prisco, G. Plizzari, L. Vandewalle, Fibre reinforced concrete: new design perspectives, Mater. Struct. 42 (2009) 1261-1281.

[7] J. Walraven, High performance fiber reinforced concrete: progress in knowledge and design codes, Mater. Struct. 42 (2009) 1247-1260.

[8] FIB, fib Model Code for Concrete Structures 2010, Ernst \& Sohn, 2013.

[9] B. Boulekbache, M. Hamrat, M. Chemrouk, S. Amziane, Flowability of fibrereinforced concrete and its effect on the mechanical properties of the material, Constr. Build. Mater. 24 (2010) 1664-1671.

[10] B. Boulekbache, M. Hamrat, M. Chemrouk, S. Amziane, Influence of yield stress and compressive strength on direct shear behaviour of steel fibre-reinforced concrete, Constr. Build. Mater. 27 (2012) 6-14.

[11] J. Dils, V. Boel, G. De Schutter, Influence of cement type and mixing pressure on air content, rheology and mechanical properties of UHPC, Constr. Build. Mater. 41 (2013) 455-463.

[12] L. Ferrara, P. Bamonte, A. Caverzan, A. Musa, I. Sanal, A comprehensive methodology to test the performance of Steel Fibre Reinforced SelfCompacting Concrete (SFR-SCC), Constr. Build. Mater. 37 (2012) 406-424

[13] L. Ferrara, N. Ozyurt, M. di Prisco, High mechanical performance of fibre reinforced cementitious composites: the role of "casting-flow induced" fibre orientation, Mater. Struct. 44 (2011) 109-128.

[14] J. Hedebratt, J. Silfwerbrand, Full-scale test of a pile supported steel fibre concrete slab, Mater. Struct. 47 (2014) 647-666.

[15] N. Ozyurt, T.O. Mason, S.P. Shah, Correlation of fiber dispersion, rheology and mechanical performance of FRCs, Cem. Concr. Comp. 29 (2007) 70-79.

[16] M. Pajek, T. Ponikiewski, Flexural behavior of self-compacting concrete reinforced with different types of steel fibers, Constr. Build. Mater. 47 (2013) 397-408.

[17] E. Parant, P. Rossi, C. Boulay, Fatigue behavior of a multi-scale cement composite, Cem. Concr. Res. 37 (2007) 264-269.

[18] P. Rossi, A. Arca, E. Parant, P. Fakhri, Bending and compressive behaviours of a new cement composite, Cem. Concr. Res. 35 (2005) 27-33.

[19] P. Rossi, O. Coussy, C. Boulay, P. Acker, Y. Malier, Comparison between plain concrete toughness and steel fibre reinforced concrete toughness, Cem. Concr. Res. 16 (1986) 303-313.

[20] L. Ferrara, M. Faifer, M. Muhaxheri, S. Toscani, A magnetic method for non destructive monitoring of fiber dispersion and orientation in steel fiber 
reinforced cementitious composites. Part 2: correlation to tensile fracture toughness, Mater. Struct. 45 (2012) 591-598.

[21] S.-T. Kang, J.-K. Kim, The relation between fiber orientation and tensile behavior in an Ultra High Performance Fiber Reinforced Cementitious Composites (UHPFRCC), Cem. Concr. Res. 41 (2011) 1001-1014.

[22] K.G. Kuder, S.P. Shah, Processing of high-performance fiber-reinforced cementbased composites, Constr. Build. Mater. 24 (2010) 181-186.

[23] X. Qian, X. Zhou, B. Mu, Z. Li, Fiber alignment and property direction dependency of FRC extrudate, Cem. Concr. Res. 33 (2003) 1575-1581.

[24] I. Sanal, N. Ozyurt Zihnioglu, To what extent does the fiber orientation affect mechanical performance?, Constr Build. Mater. 44 (2013) 671-681.

[25] F. Laranjeira, A. Aguado, C. Molins, Predicting the pullout response of inclined straight steel fibers, Mater. Struct. 43 (2010) 875-895.

[26] S.J. Barnett, J.-F. Lataste, T. Parry, S.G. Millard, M.N. Soutsos, Assessment of fibre orientation in ultra high performance fibre reinforced concrete and its effect on flexural strength, Mater. Struct. 43 (2010) 1009-1023.

[27] L. Ferrara, M. Cremonesi, N. Tregger, A. Frangi, S.P. Shah, On the identification of rheological properties of cement suspensions: rheometry, computational fluid dynamics modeling and field test measurements, Cem. Concr. Res. 42 (2012) 1134-1146.

[28] K.G. Kuder, N. Ozyurt, E.B. Mu, S.P. Shah, Rheology of fiber-reinforced cementitious materials, Cem. Concr. Res. 37 (2007) 191-199.

[29] L. Martinie, J.-F. Lataste, N. Roussel, Fiber orientation during casting of UHPFRC: electrical resistivity measurements, image analysis and numerical simulations, Mater. Struct. (2013) 1-11.

[30] L. Martinie, P. Rossi, N. Roussel, Rheology of fiber reinforced cementitious materials: classification and prediction, Cem. Concr. Res. 40 (2010) 226-234.

[31] L. Martinie, N. Roussel, Simple tools for fiber orientation prediction in industrial practice, Cem. Concr. Res. 41 (2011) 993-1000.

[32] A. Perrot, T. Lecompte, P. Estellé, S. Amziane, Structural build-up of rigid fiber reinforced cement-based materials, Mater. Struct. 46 (2013) 1561-1568.

[33] A. Ghanbari, B.L. Karihaloo, Prediction of the plastic viscosity of selfcompacting steel fibre reinforced concrete, Cem. Concr. Res. 39 (2009) 12091216.

[34] J. Kaufmann, F. Winnefeld, D. Hesselbarth, W. Trindler, Evaluation of the consistency of fiber reinforced cementitious composites, Mater. Struct. 39 (2006) 645-654.

[35] M. Li, V. Li, Rheology, fiber dispersion, and robust properties of Engineered Cementitious Composites, Mater. Struct. 46 (2013) 405-420.

[36] S.-T. Kang, J.-K. Kim, Numerical simulation of the variation of fiber orientation distribution during flow molding of Ultra High Performance Cementitious Composites (UHPCC), Cem. Concr. Comp. 34 (2012) 208-217.

[37] F. Laranjeira, A. Aguado, C. Molins, S. Grünewald, J. Walraven, S. Cavalaro, Framework to predict the orientation of fibers in FRC: a novel philosophy, Cem. Concr. Res. 42 (2012) 752-768.

[38] A. Perrot, T. Lecompte, H. Khelifi, C. Brumaud, J. Hot, N. Roussel, Yield stress and bleeding of fresh cement pastes, Cem. Concr. Res. 42 (2012) 937-944.

[39] N. Roussel, A. Lemaître, R.J. Flatt, P. Coussot, Steady state flow of cement suspensions: a micromechanical state of the art, Cem. Concr. Res. 40 (2010) 77-84.

[40] P.F.G. Banfill, Rheology of Fresh Cement and Concrete, Rheology Reviews, Chapter, 61-130, The British Society of Rheology, London, 2006.

[41] X. Chateau, G. Ovarlez, K.L. Trung, Homogenization approach to the behavior of suspensions of noncolloidal particles in yield stress fluids, J. Rheol. 52 (2008) 489-506.

[42] F. Mahaut, X. Chateau, P. Coussot, G. Ovarlez, Yield stress and elastic modulus of suspensions of noncolloidal particles in yield stress fluids, J. Rheol. 52 (2008) 287-313.

[43] F. Mahaut, S. Mokeddem, X. Chateau, N. Roussel, G. Ovarlez, Effect of coarse particle volume fraction on the yield stress and thixotropy of cementitious materials, Cem. Concr. Res. 38 (2008) 1276-1285.

[44] M. Doi, S.F. Edwards, Dynamics of rod-like macromolecules in concentrated solution. Part 1, J. Chem. Soc., Faraday Trans. 2 (74) (1978) 560-570.

[45] A.P. Philipse, The random contact equation and its implications for (colloidal) rods in packings, suspensions, and anisotropic powders, Langmuir 12 (1996). 5971-5971.
[46] A.P. Philipse, The random contact equation and its implications for (colloidal) rods in packings, suspensions, and anisotropic powders, Langmuir 12 (1996) 1127-1133.

[47] A. Poitou, F. Chinesta, G. Bernier, Orienting fibers by extrusion in reinforced reactive powder concrete, J. Eng. Mech. 127 (2001) 593-598.

[48] G.B. Jeffery, The motion of ellipsoidal particles immersed in a viscous fluid, Proc. R. Soc. Lond. A 102 (1922) 161-179.

[49] J.M. Burgers, On the motion of small particles of elongated form suspended in a viscous liquid, Second Report on Viscosity and Plasticity, Chapter 3, 16 (4) 113-184, V. Kon. Ned. Akad. Wet., (Erste Sectie), North-Holland, Amsterdam, 1938.

[50] G.K. Batchelor, Slender-body theory for particles of arbitrary cross-section in Stokes flow, J. Fluid Mech. 44 (1970) 419-440.

[51] S.M. Dinh, R.C. Armstrong, A rheological equation of state for semiconcentrated fiber suspensions, J. Rheol. 28 (1984) 207-227.

[52] S.G. Advani, C.L. Tucker, The use of tensors to describe and predict fiber orientation in short fiber composites, J. Rheol. 31 (1987) 751-784.

[53] S.G. Advani, C.L. Tucker, Closure approximations for three-dimensional structure tensors, J. Rheol. 34 (1990) 367-386.

[54] B. Souloumiac, M. Vincent, Steady shear viscosity of short fibre suspensions in thermoplastics, Rheol. Acta 37 (1998) 289-298.

[55] A.G. Gibson, S. Toll, Mechanics of the squeeze flow of planar fibre suspensions, J. Non-Newtonian Fluid Mech. 82 (1999) 1-24.

[56] J. Férec, G. Ausias, M.-C. Heuzey, P.J. Carreau, Modeling fiber interactions in semiconcentrated fiber suspensions, J. Rheol. 53 (2009) 49-72.

[57] G. Natale, M.C. Heuzey, P.J. Carreau, G. Ausias, J. Férec, Rheological modeling of carbon nanotube suspensions with rod-rod interactions, AIChE J. 60 (2014) 1476-1487.

[58] L. Onsager, The effects of shape on the interaction of colloidal particles, Ann. N Y. Acad. Sci. 51 (1949) 627-659.

[59] J.S. Cintra, C.L. Tucker, Orthotropic closure approximations for flow-induced fiber orientation, J. Rheol. 39 (1995) 1095-1122.

[60] D.A. Jack, B. Schache, D.E. Smith, Neural network-based closure for modeling short-fiber suspensions, Polym. Compos. 31 (2010) 1125-1141.

[61] J. Férec, E. Abisset-Chavanne, G. Ausias, F. Chinesta, On the use of interaction tensors to describe and predict rod interactions in rod suspensions, Rheol. Acta 53 (2014) 445-456.

[62] A. Ammar, B. Mokdad, F. Chinesta, R. Keunings, A new family of solvers for some classes of multidimensional partial differential equations encountered in kinetic theory modeling of complex fluids, J. Non-Newton. Fluid Mech. 139 (2006) 153-176.

[63] A. Ammar, B. Mokdad, F. Chinesta, R. Keunings, A new family of solvers for some classes of multidimensional partial differential equations encountered in kinetic theory modelling of complex fluids: Part II: Transient simulation using space-time separated representations, J. Non-Newton. Fluid Mech. 144 (2007) 98-121.

64] J. Férec, M. Heniche, M.C. Heuzey, G. Ausias, PJ. Carreau, Numerical solution of the Fokker-Planck equation for fiber suspensions: application to the FolgarTucker-Lipscomb model, J. Non-Newton. Fluid Mech. 155 (2008) 20-29.

[65] J.K.G. Dhont, W.J. Briels, Viscoelasticity of suspensions of long, rigid rods, Colloid Surf. A 213 (2003) 131-156.

[66] R.B. Bird, C.F. Curtiss, R.C. Armstrong, O. Hassager, Dynamics of Polymeric Liquids, Kinetic Theory, vol. 2, Wiley, New York, 1987.

[67] S. Grünewald, 9 - Fibre reinforcement and the rheology of concrete Understanding the Rheology of Concrete, Chapter, 229-256, N. Roussel, Woodhead Publishing, 2012.

[68] S. Toll, Packing mechanics of fiber reinforcements, Polym. Eng. Sci. 38 (1998) 1337-1350.

[69] M. Doi, S.F. Edwards, The Rheology of Polymer Dynamics, Oxford Science Publications, 1986

[70] S. Ranganathan, S.G. Advani, Fiber-fiber interactions in homogeneous flows of nondilute suspensions, J. Rheol. 35 (1991) 1499-1522.

[71] S. Toll, Note: on the tube model for fiber suspensions, J. Rheol. 37 (1993) 123 125. 